\title{
STRONG LIFTINGS WHICH ARE NOT BOREL LIFTINGS
}

\author{
RUSSELL A. JOHNSON
}

\begin{abstract}
The purpose of this note is to prove that any strong lifting of the circle commuting with translations cannot be a Borel lifting.
\end{abstract}

1. Introduction. In [4], A. and C. Ionescu-Tulcea proved that, if $G$ is a locally compact topological group with left Haar measure $\gamma$, then $L^{\infty}(G, \gamma)$ admits a strong lifting $\rho$ which commutes with left translations. In [7], von Neumann and Stone proved (using the continuum hypothesis) that, if $X$ is locally compact metric and $\mu$ is a positive Radon measure on $X$, then $L^{\infty}(X, \mu)$ admits a Borel lifting $\rho$ (i.e., $\rho(f)$ is a Borel function whenever $f \in L^{\infty}(X, \mu)$ ).

It is natural to ask whether a strong lifting $\rho$ on $G$ commuting with translations is a Borel lifting. We will prove that if $G=K=$ circle, then $\rho$ cannot be a Borel lifting. The proof is a simple combination of techniques of topological dynamics due to Furstenberg [3] and Veech [9].

2.

2.1. Definitions. Let $X$ be a locally compact Hausdorff space, and let $\mu$ be a positive Radon measure on $X$. Let $M^{\infty}(X, \mu)=\{f: X \rightarrow \mathbf{C} \mid f$ is bounded and $\mu$-measurable $\}$. A map $\rho: M^{\infty}(X, \mu) \rightarrow M^{\infty}(X, \mu)$ is a linear lifting of $M^{\infty}(X, \mu)$ if (i) $\rho(f)=f \mu$-a.e., (ii) $f=g$ locally $\mu$-a.e. $\Rightarrow \rho(f)=\rho(g)$ everywhere, (iii) $\rho$ is linear, (iv) $\rho(1)=1$, (v) $f>0 \Rightarrow \rho(f)>0\left(f, g \in M^{\infty}(X, \mu)\right)$. If, in addition, (vi) $\rho(f \cdot g)$ $=\rho(f) \cdot \rho(g)$ for all $f$ and $g$, then $\rho$ is a lifting. If (vii) $\rho(f)=f$ for all bounded continuous $f: X \rightarrow \mathbf{C}$, and if (i)-(vi) are satisfied, then $\rho$ is a strong lifting of $M^{\infty}(X, \mu)$.

2.2. TheOREM [4]. Let $G$ be a locally compact topological group with left Haar measure $\gamma$. There is a strong lifting $\rho$ of $M^{\infty}(G, \gamma)$ which commutes with left translations. That is, if $f \in M^{\infty}(X, \mu), g \in G$, and $(f \cdot g)(x)=_{\mathrm{def}} f(g \cdot x)(x \in G)$, then $\rho(f \cdot g)=\rho(f) \cdot g(g \in G)$.

2.3. TheOREM [7, TheOREM 17]. Let $X$ be a locally compact metric space with positive Radon measure $\mu$. Then there is a Borel lifting $\rho$ of $M^{\infty}(X, \mu)$. That is, if $f \in M^{\infty}(X, \mu)$, then $\rho(f)$ is Borel measurable.

As is remarked in [5], it is not known whether the above theorem may be proved without using the continuum hypothesis.

Received by the editors July 24, 1979.

1980 Mathematics Subject Classification. Primary 46G15, 54H20. 
2.4. Definitions and remarks. Let $K$ be the circle group, viewed as the unit circle in C. Let $\gamma$ be normalized Haar measure on $K$, and let $\rho$ be a strong lifting of $M^{\infty}(K, \gamma)$ commuting with translations. Let $\alpha_{0}$ be some irrational number and let $\alpha=e^{2 \pi i \alpha_{0}}$. Then $\left\{\alpha^{n}: n \in \mathbf{Z}\right\}$ is dense in $K$. Using a technique of Furstenberg [3, §3], we may construct a function $r: K \rightarrow K$ such that (i) $r$ is $\gamma$-measurable, but is not equal $\gamma$-a.e. to a continuous function on $K$, (ii) $r(\omega \cdot \alpha) / r(\omega)$ is equal $\gamma$-a.e. to a continuous function $q$ on $K$. See also [6]. Note that, if $\tilde{r}=\rho(r)$, then $|\tilde{r}(\omega)|=1$ and $\tilde{r}(\omega \cdot \alpha) / \tilde{r}(\omega)=q(\omega)$ for all $\omega \in \Omega$. Replacing $r$ by $\tilde{r}$, we assume from now on that $\rho(r)=r$, and that $r(\omega \cdot \alpha) / r(\omega)=q(\omega)$ for all $\omega \in \Omega$.

2.5. Definitions. Let $X$ be a compact Hausdorff space, and let $T$ be a topological group. A flow on $X$ is defined by a triple $(X, T, \eta)$, where $\eta: X \times T \rightarrow$ $X:(x, t) \rightarrow x \cdot t$ is a continuous map such that (i) $x \cdot e=x(x \in X, e=$ identity in $T)$, (ii) $\left(x \cdot t_{1}\right) \cdot t_{2}=x \cdot\left(t_{1} t_{2}\right)\left(x \in X, t_{1}, t_{2} \in T\right)$. We suppress $\eta$, and denote a flow by $(X, T)$. A subset $A$ of $X$ is invariant if $A \supset A \cdot T=\{x \cdot t \mid x \in A, t \in T\}$. A flow $(X, T)$ is minimal if each orbit $\{x \cdot t \mid t \in T\} \subset X$ is dense in $X(x \in X)$.

2.6. Definition. Let $K, r, q, \alpha$ be as in 2.4. Define a homeomorphism $h$ of $K \times K \equiv K^{2}$ as follows: $h(\omega, \zeta)=(\omega \cdot \alpha, q(\omega) \zeta)$. Then $h$ defines a flow $\left(K^{2}, Z\right)$ in the obvious way: $(\omega \cdot \zeta) \cdot n=h^{n}(\omega, \zeta)$, where $h^{n}$ is the $n$-fold composition $(n \in Z)$. (We give $\mathbf{Z}$ the discrete topology.)

The following proposition is a corollary of [1, Lemma 1.9 and Theorem 1.17].

\subsection{Proposition. The flow $\left(K^{2}, Z\right)$ defined in Definition 2.6 is minimal.}

2.8. Definition [8, Definition 0.2]. Let $(K, \mathbf{Z})$ be the flow defined by $\boldsymbol{\omega} \cdot \boldsymbol{n}=$ $\omega \cdot \alpha^{n}(\omega \in K, n \in \mathbf{Z})$. Let $(X, Z)$ be another minimal flow, suppose $\pi: X \rightarrow K$ is continuous and suppose $\pi(x \cdot n)=\pi(x) \cdot n(x \in X, n \in \mathbf{Z})$. Then $\pi$ is onto. We say $(X, Z)$ is an extension of $(K, \mathbf{Z})$. Say that $(X, Z)$ is an almost automorphic (a.a.) extension of $(K, Z)$ if $\pi^{-1}(\omega)$ is a singleton for some $\omega \in K$.

The following theorem is a special case of [9, Proposition 2.3.9].

2.9. TheOREM. Let $(X, Z)$ be an extension of $(K, Z)$ with $X$ compact metric. Suppose there is an invariant Borel subset $Q$ of $X$ such that $Q \cap \pi^{-1}(\omega)$ is a singleton for each $\omega \in K$. Then $(X, \mathbf{Z})$ is an a.a. extension of $(K, \mathbf{Z})$.

2.10. TheOREM. The strong lifting $\rho$ of $M^{\infty}(K, \gamma)$ is not a Borel lifting.

Proof. Suppose for contradiction that $\rho(f)$ is a Borel for all $f \in M^{\infty}(K, \gamma)$. Then $r=\rho(r)$ is Borel measurable. Hence the set $Q=\{(\omega, r(\omega)): \omega \in K\}$ is a Borel subset of $K^{2}$. It is also invariant under the flow $\left(K^{2}, \mathbf{Z}\right)$. By Theorem 2.9, $\left(K^{2}, Z\right)$ is an a.a. extension of $(K, Z)$. This is absurd. So $\rho(r)$ cannot be Borel.

2.11. Remarks. (1) The proof of Theorem 2.10 requires only that $\rho$ commute with multiplication by all the $\alpha^{n}(n \in \mathbf{Z})$.

(2) It is plausible to conjecture that, if $G$ and $\rho$ are as in Theorem 2.2 , then $\rho$ cannot be a Borel lifting. This statement is true if $G$ is metrizable and contains $\mathbf{Z}$ as an embedded dense subgroup. For, in this case, a function $r$ with properties (i) and (ii) of 2.4 may be constructed. 


\section{REFERENCES}

1. E. Effros and F. Hahn, Locally compact transformation groups and C*-algebras, Mem. Amer. Math. Soc. No. 75 (1967).

2. R. Ellis, Lectures on topological dynamics, Benjamin, New York, 1969.

3. H. Furstenberg, Strict ergodicity and transformations of the torus, Amer. J. Math. 83 (1961), $573-601$.

4. A. Ionescu-Tulcea and $\mathrm{C}$. Ionescu-Tulcea, On the existence of a lifting commuting with the left translations of an arbitrary locally compact group, Proc. Fifth Berkeley Sympos. Math. Statist. and Probability (Berkeley, Calif.., 1965/66), Vol. II: Contributions to Probability Theory, Part 1, Univ. of California Press, Berkeley, Calif., 1967, pp. 63-97.

5. _ Topics in the theory of lifting, Ergebnisse der Math. und ihrer Grenzgebiete, Band 48, Springer-Verlag, New York, 1969.

6. R. Johnson, The measure-theoretic structure group is not invariant, Illinois J. Math. 21 (1977), 178-184.

7. J. von Neumann and A. H. Stone, The determination of representative elements in the residual classes of a Boolean algebra, Fund. Math. 25 (1935), 353-378.

8. W. A. Veech, Point-distal flows, Amer. J. Math. 92 (1970), 205-242.

9. __ Topological dynamics, Bull. Amer. Math. Soc. 83 (1977), 775-830.

Department of Mathematics, University of Southern California, los Angeles, California 90007 